\title{
Study of the in vivo role of Mce2R, the transcriptional regulator of mce2 operon in Mycobacterium tuberculosis
}

\author{
Marina Andrea Forrellad ${ }^{1 \dagger}$, María Verónica Bianco ${ }^{1 \dagger}$, Federico Carlos Blanco ${ }^{1}$, Javier Nuñez ${ }^{2}$, Laura Inés Klepp ${ }^{1}$, \\ Cristina Lourdes Vazquez ${ }^{3}$, María de la Paz Santangelo ${ }^{1}$, Rosana Valeria Rocha ${ }^{1}$, Marcelo Soria ${ }^{4}$, Paul Golby², \\ Maximiliano Gabriel Gutierrez $z^{3,5}$ and Fabiana Bigi ${ }^{{ }^{*}}$
}

\begin{abstract}
Background: Tuberculosis is one of the leading causes of mortality throughout the world. Mycobacterium tuberculosis, the agent of human tuberculosis, has developed strategies involving proteins and other compounds called virulence factors to subvert human host defences and damage and invade the human host. Among these virulence-related proteins are the Mce proteins, which are encoded in the mce1, mce2, mce3 and mce4 operons of M. tuberculosis. The expression of the mce2 operon is negatively regulated by the Mce2R transcriptional repressor. Here we evaluated the role of Mce2R during the infection of $M$. tuberculosis in mice and macrophages and defined the genes whose expression is in vitro regulated by this transcriptional repressor.

Results: We used a specialized transduction method for generating a mce2R mutant of $M$. tuberculosis H37Rv. Although we found equivalent replication of the Mt $\Delta$ mce2R mutant and the wild type strains in mouse lungs, overexpression of Mce2R in the complemented strain (Mt $\triangle$ mce2RComp) significantly impaired its replication. During in vitro infection of macrophages, we observed a significantly increased association of the late endosomal marker LAMP-2 to Mt $\triangle$ mce2RComp-containing phagosomes as compared to Mt $\Delta$ mce2R and the wild type strains. Whole transcriptional analysis showed that Mce2R regulates mainly the expression of the mce2 operon, in the in vitro conditions studied.

Conclusions: The findings of the current study indicate that Mce2R weakly represses the in vivo expression of the mce2 operon in the studied conditions and argue for a role of the proteins encoded in Mce2R regulon in the arrest of phagosome maturation induced by M. tuberculosis.
\end{abstract}

Keywords: Mycobacterium tuberculosis, mce2R, Phagosome arresting

\section{Background}

Tuberculosis is still one of the leading causes of mortality throughout the world. The HIV/AIDS pandemic, the deterioration of public health systems in developing countries, and the emergence of multi-drug resistance of untreatable forms of tuberculosis have further contributed to that spread. Infection by the causative agent of tuberculosis, Mycobacterium tuberculosis, is achieved by strategies involving uptake and replication of the bacterium in host

\footnotetext{
*Correspondence: fbigi@cnia.inta.gov.ar

${ }^{\dagger}$ Equal contributors

'Instituto de Biotecnología, CICVyA-INTA, N. Repetto and De los Reseros, Hurlingham 1686, Argentina

Full list of author information is available at the end of the article
}

macrophages and the weakening or modification of the host immune response [1,2]. In recent years, there has been a considerable advance in the understanding of the molecular bases of pathogenicity, virulence and persistence of mycobacteria. One significant contribution to this knowledge has been the identification of essential proteins for mycobacterial virulence. The Mce (mammalian cell entry) proteins are a group of secreted or surface-exposed proteins encoded by mce genes. These genes are situated in operons, comprising eight genes, organized in exactly the same manner. M. tuberculosis has four mce loci: mce1, mce2, mce3 and mce4. The name of these proteins is derived from the function firstly assigned to Mce1, related 
to the ability of mycobacteria to enter mammalian cells and survive inside macrophages [3]. mce operons with an identical structure have been identified in all Mycobacterium species examined, as well as in other species of Actinomycetales [4]. A considerable number of studies have demonstrated that Mce proteins are related to the virulence of each member of the $M$. tuberculosis complex. Flesselles et al. [5] have reported that a BCG strain mutated in mcel exhibits a reduced ability to invade the non-phagocytic epithelial cell line HeLa. Sassetti and Rubin [6] have then found that mce1 disruption causes attenuation of $M$. tuberculosis. Further studies have shown that a strain knockout in $m c e 1$ has reduced ability to multiply when inoculated by the intratracheal route in mice. However, the same mcel mutant strain is hypervirulent when inoculated intraperitoneally in mice. Moreover, Shimono et al. [7] have demonstrated that a strain of $M$. tuberculosis mutant in the mce1 operon can kill mice more rapidly than the wild type strain after intravenous inoculation. Variations in the level of virulence depending on the route of bacterial inoculation have also been observed in mutants of the mce 2 and mce3 operons when assessed in mice [8,9], suggesting that $M$. tuberculosis regulates the expression of Mce proteins to adapt to the variety of environmental host conditions. Consistently with this presumption, regulatory proteins that control the transcription of mce1, mce 2 and mce3 have been identified in $M$. tuberculosis.

In a previous study, we have demonstrated that $m c e 2 R$ ( $R v 0586)$, the first open reading frame of the mce2 operon, encodes for a mce2-specific GntR transcriptional repressor [10]. This regulator poorly controls the expression of Mce2 proteins during the in vitro growth of M. tuberculosis in rich media [10], suggesting that Mce2R control the expression of $m c e 2$ when the bacteria encounter a particular growth-restricted environment. In order to test this possibility, in this study we compared the replication of $M$. tuberculosis in mice in the absence and in the presence of Mce2R. The genes regulated by Mce2R and the role of this regulator in the maturation of the $M$. tuberculosis-containing phagosomes in macrophages was also investigated.

\section{Results}

\section{Deletion of mce2R in $M$. tuberculosis}

The $m c e 2 R$ gene $(R v 0586)$ of $M$. tuberculosis $\mathrm{H} 37 \mathrm{Rv}$ was knocked out by replacing bases 137 to 617 of the gene with a hygromycin-resistance cassette. The integrity of the resulting $m c e 2 R$ mutant strain was then confirmed by polymerase chain reaction (PCR). Figure $1 \mathrm{~A}$ shows that no amplification product was detected in the mutant strain, with primers that hybridise within the deleted region of $m c e 2 R$, and that a product of approximately $300 \mathrm{bp}$, corresponding to the central region of $m c e 2 R$, was amplified in the wild-type strain. Using primers that hybridise $980 \mathrm{bp}$ from the $5^{\prime}$ end of $m c e 2 R$ and inside the hygromycin resistance genes, an amplicon of expected size $(1,150 \mathrm{bp})$ was detected only in the Mt $\Delta$ mce2R mutant strain. In order to evaluate the effect of the deletion in $m c e 2 R$ on the expression of mce 2 operon, changes in mRNA levels were monitored by quantitative real time PCR (RT-qPCR) in the wild type and in the Mt $\Delta$ mce2R mutant strains. Results showed a significant increase in the level of transcription of $y r b E 2 A$ and $m c e 2 A$ (Table 1) in the Mt $\Delta$ mce2R mutant strain compared to the wild type during in vitro culture $(p<0.05)$, thus confirming that Mce2R acts as a transcriptional repressor of the $m c e 2$ operon. Importantly, the reintroduction of $m c e 2 R$ significantly decreased the transcription of the mce 2 genes in the mutant strain (see below). Since our earlier work had shown that $m c e 2 R$ and the $m c e 2$ operon are cotranscribed [10], the decreased transcription of the mce 2 genes in the complemented strain further indicates that the upregulation of the $m c e 2$ gene in the knockout mutant was not the result of a polar effect of the disruption of $m c e 2 R$ but rather the consequence of a loss of repression by the regulator.

The growth profiles of the wild type, mutant and complemented strains under in vitro standard culture conditions showed similar doubling times. Thus, the mutation of the $m c e 2 R$ gene does not appear to compromise the in vitro growth of $M$. tuberculosis (data not shown).

\section{Overexpression of Mce2R reduces $M$. tuberculosis replication in a mouse model of infection}

In order to examine the infection and survival pattern of the Mt $\Delta$ mce2R mutant in vivo, we used the intratracheal route to infect $\mathrm{BALB} / \mathrm{c}$ mice [8], and determined lung colonization by counting bacterial colony forming units (CFUs). At 26 and 35 days post-infection, the number of CFUs in lungs of animals inoculated with the Mt $\Delta$ mce $2 \mathrm{R}$ mutant was equivalent than that of the animals inoculated with the parental strain (Figure 2). However, the introduction of a constitutively expressed $m c e 2 R$ gene into the $\mathrm{Mt} \Delta$ mce2R mutant (Mt $\Delta$ mce2RComp) significantly reduced the replication of $M$. tuberculosis in lungs at 26 and 35 days post-infection $(p<0.05)$. This result led us to hypothesize that the expression of the mce 2 operon was over-repressed in the complemented strain due to the overexpression of Mce2R. To test this possibility, we assessed the in vitro expression of $m c e 2 R$ and $y r b E 2 A$ in the complemented and the wild type strains at both the early and late exponential phases of bacterial growth. The level of transcription of $m c e 2 R$ in the complemented strain was higher than in the wild type strain $(p<0.05)$ at the exponential and stationary growth phases (Table 1). At the early exponential phase, the differences in the amount of $y r b E 2 A$ mRNA between both strains were 


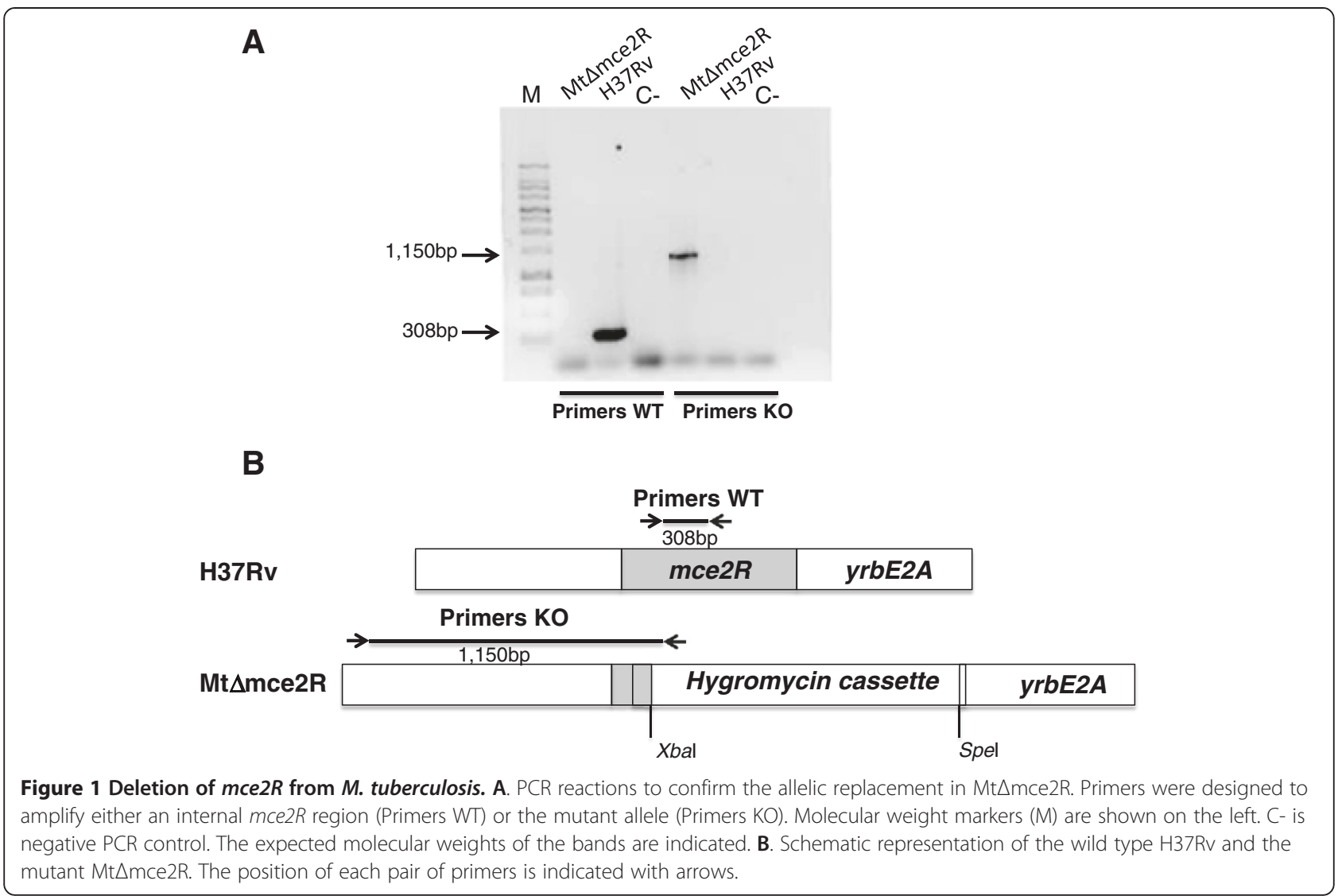

not statistically significant whereas at the late exponential phase there was a significant reduction in $y r b E 2 A$ mRNA $(p<0.05)$ in the complemented strain as compared with that in the wild type strain (Table 1).

The lack of Mce2R only affects the expression of $m$ ce 2 operon during the in vitro culture of $M$. tuberculosis To define the Mce2R regulon we performed a wholegenome in vitro expression profiling on the mutant and wild-type parental H37Rv strains. The analysis of gene expression data showed that about $99.6 \%$ of all genes showed fold changes equal or greater than 1.2 (absolute value) (Additional file 1: Table S1), indicating that most of the genes were similarly expressed in the mutant and the wild type strains. We found only 16 genes that were overexpressed in $\mathrm{Mt} \Delta$ mce2 $\mathrm{R}$ with fold changes $>1.2$.

A subset of those genes that were upregulated in the Mt $\Delta$ mce2R mutant strain in the microarray experiments was assessed by RT-qPCR. Only the mce 2 genes were significantly upregulated in the mutant strain $(p<0.05$, Table 1). The other genes that were overexpressed in the microarray experiment showed even lower and/or non-significant fold changes in the RT-qPCR assays (Additional file 1: Table S1), with the exception of $R v 0324$ that was downregulated in both the microarray and RT-qPCR experiments $(p<0.02)$. Altogether,

Table 1 Comparison of the gene expression ratios of mce2 genes, obtained by RT-qPCR

\begin{tabular}{|c|c|c|c|c|}
\hline \multirow[t]{2}{*}{ Gene name } & \multicolumn{2}{|c|}{ Fold change Mt $\Delta$ mce $2 R / H 37 R v$} & \multicolumn{2}{|c|}{ Fold change $M t \Delta m c e 2 R$ Comp/H37Rv } \\
\hline & EEP & LEP & EEP & LEP \\
\hline MtH37Rv-0587 (yrbE2A) & 4.95 & ND & $-2.71^{*}$ & -5.43 \\
\hline MtH37Rv-0586 (mсе2R) $\Psi$ & 10.14 & 3.47 & 29.5 & 3.99 \\
\hline MtH37Rv-0589 (mce2A) & 6 & ND & ND & ND \\
\hline MtH37Rv-0590 (mce2B) & ND & ND & ND & -4.6 \\
\hline
\end{tabular}

*Values were not statistically different between strains.

UPrimers encompass $137 \mathrm{bp}$ of the $5^{\prime}$ end of Rv0586, which are conserved in the mutant.

Abbreviations: ND not determined, EEP early exponential phase, LEP late exponential phase.

The values indicate the average ratios of $\mathrm{Mt} \Delta \mathrm{mce} 2 \mathrm{R} / \mathrm{M}$. tuberculosis $\mathrm{H} 37 \mathrm{Rv}$ or Mt $\Delta$ mce2R Comp/M. tuberculosis H37Rv for four independent biological replicates. 


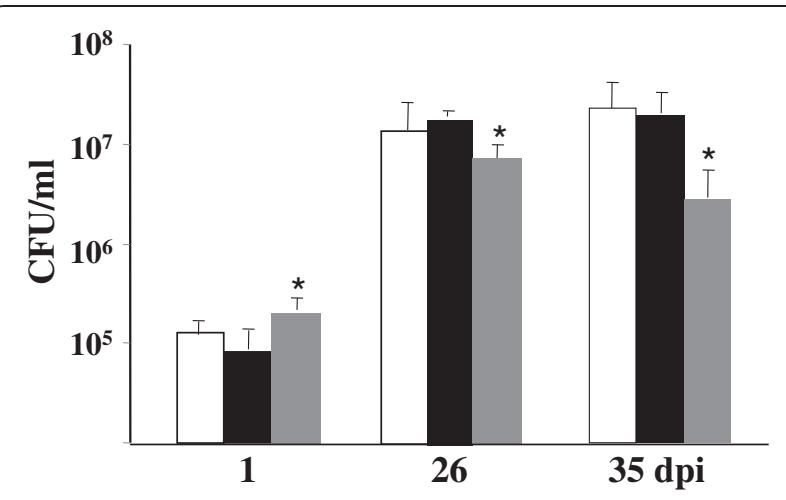

Figure 2 Replication of the Mt $\Delta$ mce2R mutant, the wild type and complemented strains in mouse lungs after intratracheal inoculation. Groups of mice were infected by intratracheal injection of wild type (white bars), Mt $\Delta$ mce $2 R$ (black bars), Mt $\Delta$ mce2RComp (grey bars). At 1, 26 and 35 days post-infection, mice were sacrificed and viable bacteria present in the lungs were recovered. The results are expressed as the mean number of CFUs \pm standard deviations in five mice. These data are based on one of two independent experiments with similar results. ${ }^{*}(p<0.05)$ significantly different from values of the wild type strain.

these results indicate that in standard in vitro culture conditions Mce2R mainly regulates the expression of the mce 2 operon.

\section{Overexpression of $m c e 2 R$ reduces the arrest of mycobacteria-containing phagosomes}

We next evaluated the maturation stage of mycobacterial phagosomes using immunofluorescence and confocal microscopy. M. tuberculosis strains were used to infect J774 macrophages for 1 hour of uptake and two hours of chase as described in Material and Methods and processed for microscopy.

In three of four independent experiments, the fraction of Lysosomal-associated membrane protein 2 (LAMP-2)positive phagosomes was slightly, but significantly $(p<$ 0.01 ), lower in cells infected with Mt $\Delta$ mce $2 \mathrm{R}$, as compared to the wild-type strain (Figure 3 ). Consistently with the in vivo replication experiments, overexpression of Mce2R in the complemented strain significantly increases the maturation of $M$. tuberculosis-containing phagosome $(p<0.001)$. These results suggest that Mce2R regulon participates in the phagosomal arrest induced by intracellular M. tuberculosis to survive and replicate inside macrophages [11]. In order to know the contribution of mce 2 operon to the phagosome arresting we evaluated the association of LAMP-2 marker with phagosomes containing a M. tuberculosis mce2-knockout (Mt $\Delta$ mce2, [8]). In two independent experiments the number of LAMP-2-positive phagosomes were higher $(p<0.001)$ in cells infected with Mt $\Delta$ mce 2 than in those infected with the wild-type strain (Figure 3), indicating that mce 2 operon encodes proteins with a role in phagosome arresting.

\section{Discussion}

In the present study we demonstrated that the knockout of the transcriptional repressor Mce2R does not affect the replication of $M$. tuberculosis in mouse lungs. This finding suggests that similarly to that observed for the in vitro growth of $M$. tuberculosis, Mce2R weakly represses the in vivo expression of the mce2 virulence operon, likely due to the fact that this repressor negatively regulates its own expression. Remarkably, when the transcription of $m c e 2 R$ was conducted by a strong and desregulated promoter, the resulting complemented strain expressed higher levels of $m c e 2 R$ mRNA than the wild type strain, and was significantly more attenuated than the mutant $M$. tuberculosis strain, in terms of bacterial replication in lungs. Thus, these observations may indicate that, during the in vivo infection, the expression of the mce 2 operon is more effectively repressed in the complemented strain than in the wild type strain. In in vitro growth conditions, the expression of $y r b E 2 A$ was significantly repressed in the complemented strain only at the stationary growth phase, suggesting that Mce2R could effectively repress the transcription of the mce 2 operon when a substantial level of this repressor is accumulated. This in vitro mce 2 expression profile supports the hypothesis that increasing bacterial attenuation along the infection is a consequence of an increasing reduction of the expression of the mce 2 operon. Importantly, the results of this study are consistent with previous findings demonstrating that a mutation in the mce 2 operon impairs either the replication or the lethality of $M$. tuberculosis in mouse models [8,9].

We also defined the in vitro Mce2R regulon by whole genome microarray analysis and determined that the genes whose expressions were significantly affected by the transcriptional regulator were confined to those belonging to the $m c e 2$ operon. Surprisingly, the expression of the end gene, which has been suggested to be regulated by Mce2R [10], showed no changes in expression in the mutant strain compared to the wild type. This difference is probably a reflection of the different experimental setups in each study. While in the present study the conditions used to study gene expression were based on the absence or presence of Mce2R, our previous study investigated the effect of modulating the expression of $m c e 2 R$. The expression $R v 0324$, which encodes a putative transcriptional regulator, was slightly reduced in the mutant strain, suggesting that the lack of Mce2R indirectly affects the expression of $R v 0324$. However, the low fold change detected for this gene in both experimental strategies places in doubt the biological significance of this differential expression. The type of exclusive in vitro regulation of Mce2R over the mce2 operon contrasts to that described for Mce3R, the transcriptional repressor of the mce3 operon $[12,13]$. Whereas during the in vitro 

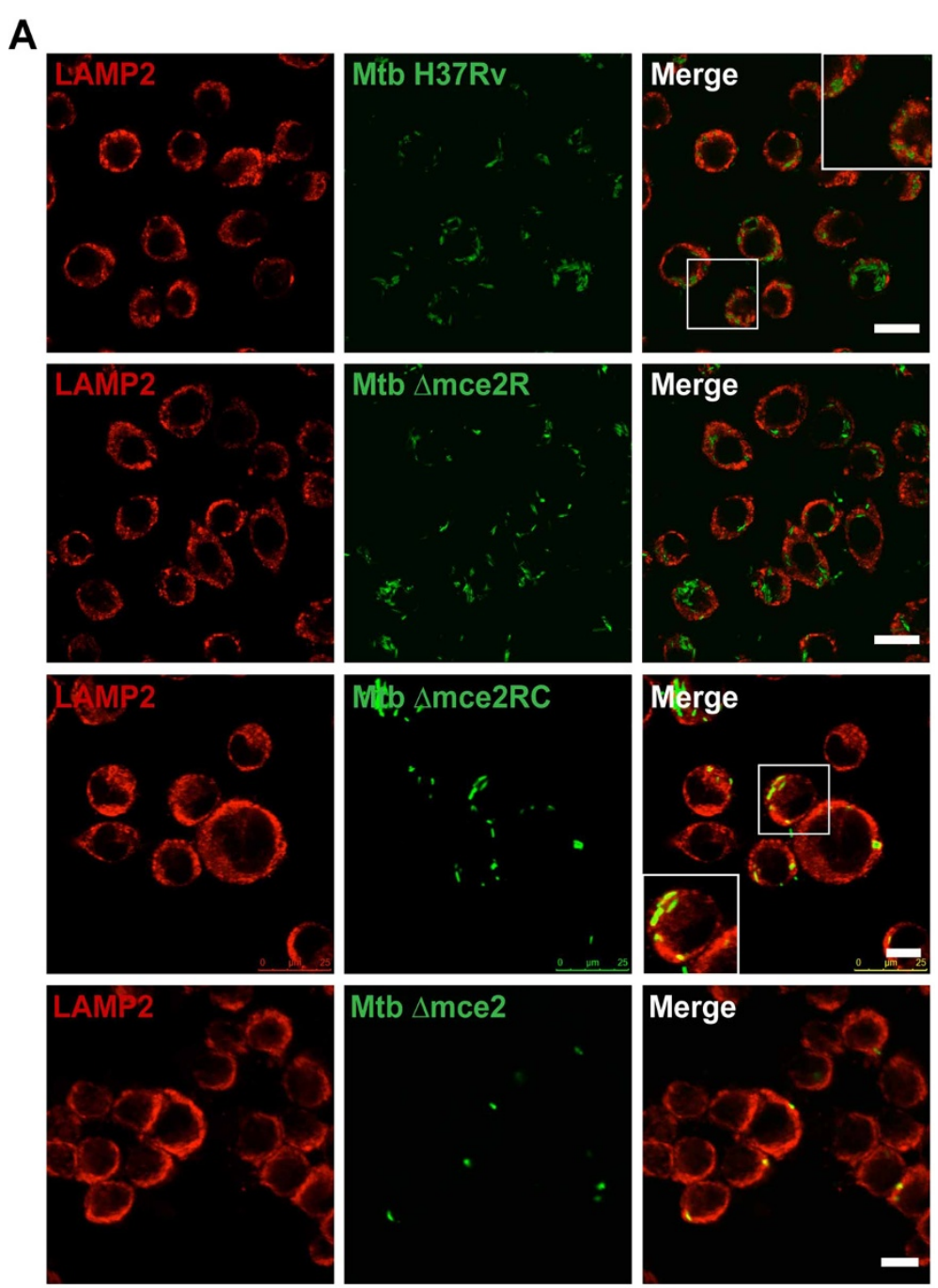

B

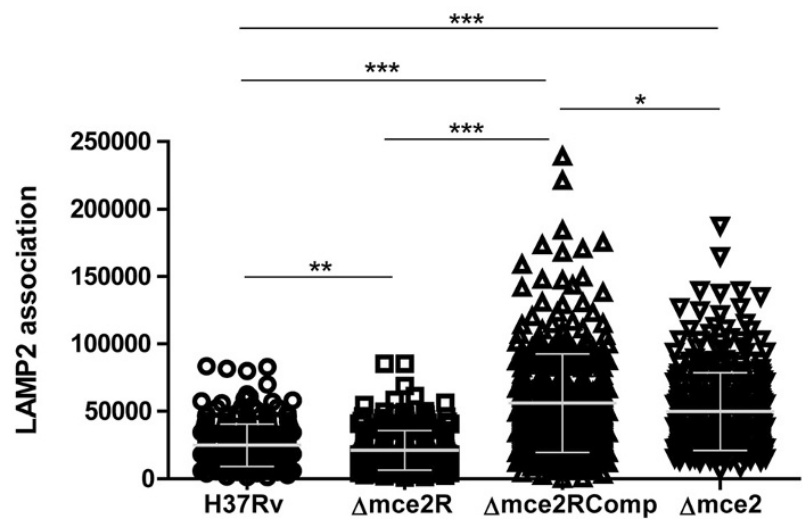

Figure 3 The overexpression of $m c e 2 R$ decreases the arrest of phagosome maduration. A. LAMP-2 association of M. tuberculosis H37RV, Mt $\Delta$ mce2R, Mt $\Delta$ mce2RComp and Mt $\Delta$ mce2-containing phagosome. J774 macrophages were infected with M. tuberculosis strains for 1 h, washed and incubated for additional $2 \mathrm{~h}$ in RPMI media. Phagosomal LAMP-2 was detected using an appropriate antibody (red) and the bacteria were stained with FITC (green). The cells were analyzed by confocal microscopy and in the Merge box is observed the bacteria-LAMP2 association. Scale bars: $10 \mu \mathrm{m}$. B. Quantification of that observed in A). These data are based on one of two-four independent experiments with similar results. $\left(^{* *}\right)$ Indicates significance where $p<0.001,\left({ }^{* *}\right)$ where $p<0.01$ and $\left(^{*}\right)$ where $p<0.05$. 
growth of $M$. tuberculosis, Mce3R negatively regulates the expression of two transcriptional units likely to be involved in lipid or isoprenoid modifications [13], Mce2R seems to regulate exclusively the transcription of $m c e 2$. Our findings thus suggest that the higher virulence of the mutant Mt $\Delta$ mce $2 R$ strain (as compared to the $m c e 2 R$ overexpressing-complemented strain) is, at least in part, a consequence of the overexpression of the mce 2 operon.

One of the main mechanisms elicited by intracellular mycobacteria to survive and replicate inside the host cells is to arrest the normal process of phagosome maturation, which enables bacterial survival in a non-acidified intracellular compartment [11]. Proteins involved in the biosynthesis of cell wall lipids, such as PhoP [14] and Ag85A [15], have shown to have a role in the phagosome arresting exerted by M. tuberculosis. Likely, these proteins are not direct modulators of phagosome trafficking, instead they would participate in the synthesis of compounds that are actually implicated in this cellular process. For instance, the synthesis of cell wall trehalose dimycolate and the sulfolipids is regulated by the twocomponent system PhoP/PhoR and these lipids have been described as implicated in blocking phagosome/lysosome fusion induced by $M$. tuberculosis [11]. However, a recent report has suggested the opposite, showing that overproduction of the sulfoglycolipids (SGL), Ac3SGL and Ac4SGL in the M. tuberculosis Rv1503c::Tn and Rv1506c:: Tn strains increases the intracellular trafficking to lysosomes of these mutant strains. In connection with this last finding, previous reports have suggested a role of the proteins encoded in the $m c e 2$ operon in the sulpholipid metabolism/transport. Firstly, Marjanovic et al. have shown that a $M$. tuberculosis deleted in mce 2 operon accumulates more sulpholipids (SLs) than it parental $\mathrm{H} 37 \mathrm{Rv}$ strain, proposing that the mce 2 operon encodes proteins involved in the metabolism/transport of SLs [16]. Secondly, the finding that sigma factor $\mathrm{L}$ seems to regulate the expression of mce 2 genes and genes encoding enzymes implicated in SL synthesis and the fact that the mce 2 operon is absent in Mycobacterium smegmatis [4], which does not produce SL-1 [17], also support a role of Mce2 proteins in the transport of SLs. Based on these previous observations and the results of this study, we can speculate that lack of Mce2 proteins (either by mutation or overrepression) increases the accumulation of SLs in the bacteria, disfavouring the arrest of phagosome maturation and in turn the survival of both the mutant Mt $\Delta$ mce2 [8] and the complemented Mt $\Delta$ mce2Comp in mouse lungs. However, the higher maturation of phagosomes containing the over-repressed strain (Mt $\Delta$ mce2RComp) as compared to that of phagosomes containing Mt $\Delta$ mce $2(p<0.05)$ may indicate that other in vivo Mce2R-regulated genes can also participate in the phagosome arresting induced by intracellular M. tuberculosis. Whether the mutation of mce2R affects the accumulation of SLs in M. tuberculosis will require further investigation and is beyond the scope of the present study.

\section{Conclusions}

The results of the present study demonstrate that, in standard conditions of in vitro culture, Mce2R mainly regulates the expression of the $m c e 2$ operon and support a role of the proteins regulated by this transcriptional repressor in the virulence of $M$. tuberculosis. In addition, we showed that this role in the virulence for Mce2R regulon takes place in part through interfering with the normal maturation of phagosomes. However, further research is needed to better understand the mechanisms of regulation exerted by Mce $2 \mathrm{R}$ and the role of Mce2R regulon in the survival of $M$. tuberculosis inside the host.

\section{Methods}

\section{Ethical statement}

Animal experimentations were performed inside the biosafety facilities of the National Institute of Agricultural Technology (INTA), Argentina, in compliance with the regulations of Institutional Animal Care and Use Committee (CICUAE) of INTA (file number: 31-20-12). CICUAE's members: Florestán Maliandi (President), Alejandra Romera (Secretary), Marisa Farber, Analía Berinstein, Pablo Chacana, Gabriel Pinto, Bibiana Brihuega, Gisella Marcoppido, Verónica Maldonado May, Lucas Vagnoni, Osvaldo Zabal and Luis Samartino (Vocals).

\section{Bacterial strains and culture media}

All cloning steps were performed in Escherichia coli HB101. E. coli were grown either in Luria-Bertani (LB) broth or on LB agar. $M$. tuberculosis strains were grown in Middlebrook 7H9 medium supplemented with albumin $0.5 \%$, dextrose $0.4 \%$, and glycerol $0.5 \%$ (M7H9AD-G) and either Tween $800.05 \%$ or Middlebrook 7H11, supplemented with albumin, dextrose and glycerol. When necessary, either $50 \mu \mathrm{g} / \mathrm{ml}$ hygromycin or $20 \mu \mathrm{g} / \mathrm{ml}$ kanamycin was added to the media.

\section{Construction of $M$. tuberculosis $\Delta$ mce $2 R$ mutant and complemented strains}

A mutant strain of $M$. tuberculosis carrying a chromosomal deletion encompassing the bases 137-617 of the $m c e 2 R$ ( $R v 0586$ ) gene was obtained by using the gene knockout system described by Bardarov [18]. Briefly, two DNA fragments of approximately $1 \mathrm{~kb}$ flanking the $5^{\prime}$ and 3 ' regions of $m c e 2 R$ were obtained by PCR using M. tuberculosis $\mathrm{H} 37 \mathrm{Rv}$ genomic DNA as template and the following sets of primers: Regionup-up (tctagaccgta caactcgatcaat)/Regionup-low (tctagaactccgagcaactcagcc) and Regionlow-up (actagtatctgctcaggtgatccc)/Regionlow-low (actagtacgccgatcgtggtcaac). Flanking arms were directionally 
cloned into XbaI and SpeI sites of cosmid pYUB854 [14]. The recombinant cosmid was digested by PacI and ligated to PacI-digested concatemerized DNA of phage phAE87. To generate each specialized transducing phage, the PacIdigested recombinant cosmid was used to replace cosmid pYUB328 in phAE87 an in vitro $\lambda$-packaging reaction (GIGAPackII, Stratagene). After transducing E. coli HB101 and plating the transductants on selective media containing hygromycin. Phasmid DNA was prepared from the pooled antibiotic-resistant transductants and electroporated into M. smegmatis $\mathrm{mc}^{2} 155$. Transductants were grown at the permissive temperature of $31^{\circ} \mathrm{C}$ to allow phage replication, and then transducing phages were prepared from isolated plaques as previously described [18]. Transducing phages, carrying the mutated allele of $m c e 2 R$ were used to infect $M$. tuberculosis H37Rv as previously described [18]. Infected mycobacteria were plated onto media containing hygromycin at the restrictive temperature of $37^{\circ} \mathrm{C}$. Colonies that appeared after 25 days of culturing were analysed by PCR for the presence of the deletion in the $m c e 2 R$ gene. Only one clone showed a 480-bp deletion from $m c e 2 R$ and was referred to as $\mathrm{Mt} \Delta \mathrm{mce} 2 \mathrm{R}$. Deletion of $m c e 2 R$ in $\mathrm{Mt} \Delta \mathrm{mce} 2 \mathrm{R}$ strain was confirmed by PCR analysis using two sets of primers: one set that hybridises inside $m c e 2 R$ (WT-forward: gatctgttg ccccgattgt/WT-reverse: tctacgatcgaaccggcgct), and the other that hybridises at approximately $1000 \mathrm{bp}$ from the 5 ' ends of both $m c e 2 R$ and inside the hygromycin resistance gene (KO-forward [Low new2R] acgtcagcttcagcca gagt, KO-reverse [5'hygro-reverse]: tcagcaacaccttcttcacg).

In order to complemente the mutant phenotype, a fragment containing $m c e 2 R$ gene was amplified by PCR using the primers up mce2r pw16 (catatgatctgttgccc cgattgttgt) and low mce2r pw16 (catatgcattgccgactcgcct), and cloned into $\mathrm{pW} 16$ to produce plasmid $\mathrm{pW} 16$ mce2R. This plasmid was used to transform the $M$. tuberculosis $\mathrm{Mt} \Delta$ mce2R strain by electroporation to produce the complemented strain Mt $\Delta$ mce2RComp.

\section{Mouse infections}

The experimental BALB/c model of progressive pulmonary tuberculosis has been previously described in detail [8]. Briefly, bacillary suspensions were adjusted to $1.25 \times 10^{5}$ viable cells in $100 \mu \mathrm{l}$ phosphate buffer saline (PBS). Each animal was anesthetized and intratracheally inoculated with $M$. tuberculosis strains. Infected mice were kept in cages fitted with microisolators connected to negative pressure. Groups of 15 mice were each infected with the different $M$. tuberculosis strains. The inoculum doses were determined by enumerating the CFUs recovered from the lungs of five mice per infection strain $24 \mathrm{~h}$ post-infection. Five mice per group were killed at 1,26 and 35 days after infection and lungs removed and homogenized. Four dilutions of each homogenate were spread onto duplicate plates. This experiment was repeated twice with similar results. Animal experimentations were performed inside the biosafety facilities of the National Institute of Agricultural Technology (INTA), Argentina, in compliance with the regulations of Institutional Animal Care and Use Committee (CICUAE) of INTA. Student's $t$ test was used to determine significant differences between groups.

\section{Macrophage infections}

M. tuberculosis strains were cultivated until exponential growth phase, pelleted, washed twice in PBS and resuspended in RPMI medium to a multiplicity of infection (m.o.i.) of 5. Clumps were removed by ultrasonic treatment in a water bath followed by a low speed centrifugation for $2 \mathrm{~min}$. Macrophages were seeded into 24 well tissue culture plates at $80 \%$ confluence and infected for 1 hour (uptake). Afterwards, cells were washed and incubated in full medium for another 2 hours (chase).

\section{Inmunofluorescense and confocal microscopy}

For indirect immunofluorescence, $M$. tuberculosis strains treated as described above, were covalently stained with FITC (isomer I; Sigma. FITC solution was prepared $20 \mathrm{mg} / \mathrm{ml}$ in DMSO). Briefly, $1 \times 10^{9}$ bacteria were washed twice with $0.1 \mathrm{M}$ buffer $\mathrm{Na}_{2} \mathrm{CO}_{3} / \mathrm{NaHCO}_{3}$ $(\mathrm{pH} 9)$ and suspended in $1 \mathrm{ml}$ of the same solution. FITC was added to a final concentration of $1 \mathrm{mg} / \mathrm{ml}$ and incubated in the dark for $2 \mathrm{~h}$ at $37^{\circ} \mathrm{C}$. Bacteria were washed gently with PBS until unbound colorant was eliminated, and used to infect J774 macrophages as was described above. Infected cells were fixed with $3 \%$ paraformaldehyde solution in PBS for 20 min and quenched by incubating with $50 \mathrm{mM}$ glycine solution for $10 \mathrm{~min}$. Then, cells were permeabilized with $0.05 \%$ saponin in PBS containing $0.2 \%$ BSA for $15 \mathrm{~min}$, and incubated with the primary anti-LAMP-2 (ABL-93, DSHB) antibodies diluted 1:50 in PBS. anti-LAMP-2 antibodies were obtained from the Developmental Studies Hybridoma Bank, developed under the auspices of the NICHD and maintained by The University of Iowa, Department of Biology, Iowa City, IA 52242. Secondary antibodies anti-Rat Cy5-conjugated (Jackson Immuno Research Labs Inc.) was used diluted 1:600 in PBS. Each step with antibodies was incubated for 1 hour. Cells were mounted with Dako mounting media (Dako, Denmark) and analysed by confocal microscopy using a Leica SP5 AOBS confocal microscope (Leica Microsystems, Germany). Internalization of the mycobacteria was followed through the fluorescence of green FITC and the LAMP-2 association to mycobacterial phagosomes was counted in at least 50 cells using Fiji/ ImageJ program (U.S. National Institute of Health, Bethesda, Maryland, USA). The analysis was performed for duplicates in three-four independent experiments. Statistical determinations were made using $t$ test. 


\section{RNA preparation}

DNA-free RNA was extracted from $50 \mathrm{ml}$ mid-exponentialphase cultures of $M$. tuberculosis as described by Santangelo et al. (2002) [12].

Prehybridisation, hybridisation, and washing steps were performed as described previously [13,19]. Microarrays were hybridised with a combination of Cy3-cDNA generated from genomic DNA of M. tuberculosis H37Rv and Cy5-cDNA obtained from total RNA of either $M$. tuberculosis $\mathrm{H} 37 \mathrm{Rv}$ or $\mathrm{Mt} \Delta \mathrm{mce} 2 \mathrm{R}$.

Eight sets of microarray data, consisting of eight biological replicates (cells from independent cultures), were produced for each $M$. tuberculosis strain.

The microarrays were scanned using an Affymetrix 428 scanner and fluorescent spot intensities were quantified using BlueFuse for Microarrays v3.2 (BlueGnome Limited, www.cambridgebluegnome.com). For each spot, background fluorescence was subtracted from the average spot fluorescence to produce a channel specific ratio.

\section{Data processing and statistical analysis}

Log2 Cy5:Cy3 (test:control) ratios were used for subsequent calculations. Within each microarray, block median normalisation, excluding control and empty spots, was carried out using the BlueFuse software. Median absolute deviation using Mathematica 5.2 (Wolfram Research Inc.) was applied to bring the histograms of all microarrays into the same scale. Technical replicates were averaged. Differentially expressed genes between the strains were detected by applying t-tests with a Benjamini and Hochberg adjusted pvalue correction.

\section{RT-qPCR}

RT-qPCR reactions were performed as described by Santangelo et al. $[13,20]$ using DNA-free RNA $(1 \mu \mathrm{g})$ extracted from mid-exponential growth-phase cultures and specific primers. Relative quantification was performed by using $\operatorname{sig} A$ as a reference gene and a subsequent analysis for statistical significance of the derived results was performed by using the Pair Wise Fixed Reallocation Randomization test [21]. The mean value of PCR efficiency for the primers (Additional file 2: Table S2) was $92 \%$ to $100 \%$. These values were calculated using both the classical dilution curve and slope calculation $(E=10[-1 /$ slope $]-1)[21]$ and an estimation by absolute fluorescence increase [22].

\section{Additional files}

Additional file 1: Table S1. Differential expressed genes between Mt $\triangle$ mce2R/M. tuberculosis H37Rv.

Additional file 2: Table S2. Primers used in RT-qPCR.

\section{Competing interests}

The authors declare that they have no competing interests.

\section{Authors' contributions}

MAF carried out the confocal studies and immunoassays, and drafted the manuscript. MVB carried out the infections, FCB carried out the RT-qPCR, JN and MS carried out the statistical analysis, MPS constructed the mutant strain, RVR constructed the complemented strain, CLV and MGG participated in the design of the study, PG and LIK performed the microarray study analysis, FB conceived of the study, and participated in its design and coordination and helped to draft the manuscript. All authors read and approved the final manuscript.

\section{Acknowledgements}

We acknowledge The Wellcome Trust for funding BuG@S (Bacterial Microarray Group at St George's, University of London) for supply of the microarray and associated support. We are grateful to Julia Sabio y García for her technical assistance in the confocal experiments. We also thank the group of Dr. Jacobs Jr WR for the specialized transduction system provided. The present study was supported by NIH/NIAID 1R01AI083084. Experiments with animals were funded by INTA grant PE PNBIO 1131034 and ANCyPT grant PICT 1103. MP Santangelo and F. Bigi are CONICET fellows. FB and MGG are supported by a cooperation grant from Ministry of Science and Technology (MinCyT-Argentina) and International Buro of the Federal Ministry of Education and Research (Germany).

\section{Author details}

${ }^{1}$ Instituto de Biotecnología, CICVyA-INTA, N. Repetto and De los Reseros, Hurlingham 1686, Argentina. ${ }^{2}$ Animal Health Veterinary Laboratories Agency (Weybridge), New Haw, Addlestone, Surrey KT15 3NB, UK. ${ }^{3}$ Research Group Phagosome Biology, Helmholtz Centre for Infection Research, Inhoffenstrasse 7, Braunschweig 38124, Germany. ${ }^{4}$ Microbiología Agrícola Facultad de Agronomía, Universidad de Buenos Aires, Avenue San Martín 4453, Buenos Aires 1417, Argentina. ${ }^{5}$ Present address: Division of Mycobacterial Research, MRC National Institute for Medical Research, The Ridgeway, Mill Hill, London NW7 1AA, UK

Received: 12 April 2013 Accepted: 29 August 2013

Published: 5 September 2013

\section{References}

1. Glickman MS, Jacobs WR Jr: Microbial pathogenesis of Mycobacterium tuberculosis: dawn of a discipline. Cell 2001, 104:477-485.

2. Hingley-Wilson SM, Sambandamurthy VK, Jacobs WR Jr: Survival perspectives from the world's most successful pathogen, Mycobacterium tuberculosis. Nat Immunol 2003, 4:949-955.

3. Arruda S, Bomfim G, Knights R, Huima-Byron T, Riley LW: Cloning of an M. tuberculosis DNA fragment associated with entry and survival inside cells. Science 1993, 261:1454-1457.

4. Casali N, Riley LW: A phylogenomic analysis of the Actinomycetales mce operons. BMC Genomics 2007, 8:60.

5. Flesselles B, Anand NN, Remani J, Loosmore SM, Klein MH: Disruption of the mycobacterial cell entry gene of Mycobacterium bovis BCG results in a mutant that exhibits a reduced invasiveness for epithelial cells. FEMS Microbiol Lett 1999, 177:237-242.

6. Sassetti CM, Rubin EJ: Genetic requirements for mycobacterial survival during infection. Proc Natl Acad Sci U S A 2003, 100:12989-12994.

7. Shimono N, Morici L, Casali N, Cantrell S, Sidders B, et al: Hypervirulent mutant of Mycobacterium tuberculosis resulting from disruption of the mce1 operon. Proc Natl Acad Sci U S A 2003, 100:15918-15923.

8. Gioffre A, Infante E, Aguilar D, Santangelo MP, Klepp L, et al: Mutation in mce operons attenuates Mycobacterium tuberculosis virulence. Microbes Infect 2005, 7:325-334

9. Marjanovic O, Miyata T, Goodridge A, Kendall LV, Riley LW: Mce2 operon mutant strain of Mycobacterium tuberculosis is attenuated in C57BL/6 mice. Tuberculosis (Edinb) 2010, 90:50-56.

10. Santangelo Mde L, Blanco F, Campos E, Soria M, Bianco MV, et al: Mce2R from Mycobacterium tuberculosis represses the expression of the mce 2 operon. Tuberculosis (Edinb) 2009, 89:22-28.

11. Rohde K, Yates RM, Purdy GE, Russell DG: Mycobacterium tuberculosis and the environment within the phagosome. Immunol Rev 2007, 219:37-54. 
12. Santangelo MP, Blanco FC, Bianco MV, Klepp LI, Zabal O, et al: Study of the role of Mce3R on the transcription of mce genes of Mycobacterium tuberculosis. BMC Microbiol 2008, 8:38.

13. de la Paz SM, Klepp L, Nunez-Garcia J, Blanco FC, Soria M, et al: Mce3R, a TetR-type transcriptional repressor, controls the expression of a regulon involved in lipid metabolism in Mycobacterium tuberculosis. Microbiology 2009, 155:2245-2255.

14. Ferrer NL, Gomez AB, Neyrolles O, Gicquel B, Martin C: Interactions of attenuated Mycobacterium tuberculosis phoP mutant with human macrophages. PLoS One 2010, 5:e12978.

15. Katti MK, Dai G, Armitige LY, Rivera Marrero C, Daniel S, et al: The Delta fbpA mutant derived from Mycobacterium tuberculosis H37Rv has an enhanced susceptibility to intracellular antimicrobial oxidative mechanisms, undergoes limited phagosome maturation and activates macrophages and dendritic cells. Cell Microbiol 2008, 10:1286-1303.

16. Marjanovic O, lavarone AT, Riley LW: Sulfolipid accumulation in Mycobacterium tuberculosis disrupted in the mce2 operon. J Microbiol 2011, 49:441-447.

17. Rivera-Marrero CA, Ritzenthaler JD, Newburn SA, Roman J, Cummings RD: Molecular cloning and expression of a novel glycolipid sulfotransferase in Mycobacterium tuberculosis. Microbiology 2002, 148:783-792.

18. Bardarov S, Bardarov S Jr, Pavelka MS Jr, Sambandamurthy V Jr, Larsen M, et al: Specialized transduction: an efficient method for generating marked and unmarked targeted gene disruptions in Mycobacterium tuberculosis, M. bovis BCG and M. smegmatis. Microbiology 2002, 148:3007-3017.

19. Blanco FC, Nunez-Garcia J, Garcia-Pelayo C, Soria M, Bianco MV, et al: Differential transcriptome profiles of attenuated and hypervirulent strains of Mycobacterium bovis. Microbes Infect 2009, 11:956-963.

20. Blanco FC, Soria M, Bianco MV, Bigi F: Transcriptional response of peripheral blood mononuclear cells from cattle infected with Mycobacterium bovis. PLOS ONE 2012, 7(7):e41066. doi:10.1371/journal.pone.0041066.

21. Pfaffl MW, Horgan GW, Dempfle L: Relative expression software tool (REST) for group-wise comparison and statistical analysis of relative expression results in real-time PCR. Nucleic Acids Res 2002, 30:e36.

22. Ramakers C, Ruijter JM, Deprez RH, Moorman AF: Assumption-free analysis of quantitative real-time polymerase chain reaction (PCR) data. Neurosci Lett 2003, 339:62-66.

doi:10.1186/1471-2180-13-200

Cite this article as: Forrellad et al: Study of the in vivo role of Mce2R, the transcriptional regulator of mce2 operon in Mycobacterium tuberculosis. BMC Microbiology 2013 13:200.

\section{Submit your next manuscript to BioMed Central and take full advantage of:}

- Convenient online submission

- Thorough peer review

- No space constraints or color figure charges

- Immediate publication on acceptance

- Inclusion in PubMed, CAS, Scopus and Google Scholar

- Research which is freely available for redistribution 